\title{
Verificação do conhecimento dos profissionais quanto ao mnemônico FAST HUG na unidade de terapia intensiva no hospital do norte brasileiro
}

\author{
Verification of the knowledge of professionals regarding the FAST HUG mnemonic in the intensive \\ care unit of the hospital in northern Brazil \\ Verificacíon del conocimiento de los profesionales sobre el mnemónico FAST HUG en la unidad de \\ cuidados intensivos del hospital del norte de Brasil
}

Recebido: 18/11/2021 | Revisado: 24/11/2021 | Aceito: 25/11/2021 | Publicado: 05/12/2021

\author{
Thais Dias Zumack \\ ORCID: https://orcid.org/0000-0002-5356-4956 \\ Hospital Regional de Cacoal, Brasil \\ E-mail: thais_zumack93@hotmail.com \\ Elissa Gonçalves de Oliveira e Silva \\ ORCID: https://orcid.org/0000-0001-8022-4511 \\ Hospital Regional de Cacoal, Brasil \\ E-mail: elissas_br@yahoo.com.br \\ Valcione da Silva Gretzler \\ ORCID: https://orcid.org/0000-0001-9808-7201 \\ Hospital Regional de Cacoal, Brasil \\ E-mail: valcionegretzler18@gmail.com \\ Danielle Maesima Saito \\ ORCID: https://orcid.org/0000-0001-7735-0066 \\ Hospital Regional de Cacoal, Brasil \\ E-mail: danisaito30@hotmail.com \\ Simone Rosária Soares de Moraes Cunha \\ ORCID: https://orcid.org/0000-0002-1105-7868 \\ Hospital Regional de Cacoal, Brasil \\ E-mail: Simone.rsmc@gmail.com \\ Francisca Letícia Moreira Lustosa Portela \\ ORCID: https://orcid.org/0000-0002-6053-8115 \\ Hospital Regional de Cacoal, Brasil \\ E-mail: leticiaportella@gmail.com
}

\begin{abstract}
Resumo
Trata esse estudo de verificar o conhecimento dos profissionais de saúde do SUS, médicos, enfermeiros e fisioterapeutas, que atuam na Unidade de Terapia Intensiva de um hospital público da região norte do Brasil, sobre o mnemônico FAST HUG. Foi realizado um estudo do tipo transversal e descritivo, com avaliação qualitativa e quantitativa, de amostra do tipo probabilística. A coleta de dados foi realizada por meio de questionário online com questões objetivas aprovado pelo comitê de ética e pesquisa- CEP. Dos 50 profissionais da UTI contatados apenas 25 se dispuseram a responder à consulta. Desses $52 \%$ afirmaram conhecer o checklist FAST HUG. O melhor momento para aplicação do protocolo mostrou ser durante os Rounds. Outra informação relevante constatada mostrou que $40 \%$ dos profissionais apresentaram dificuldades em aplicar os itens do checklist evidenciando a necessidade de treinamento para alinhamento de ideias e condução dos trabalhos. A instituição dos Rounds nas UTIs se mostra de uma utilidade imprescindível para a aplicação do mnemônico FAST HUG.
\end{abstract}

Palavras-chave: Protocolos clínicos; Unidade de Terapia Intensiva; Multiprofissional; Ckecklist; Bundles.

\begin{abstract}
This study aims to verify the knowledge of SUS health professionals, physicians, nurses and physiotherapists, who work in the Intensive Care Unit of a public hospital in northern Brazil, about the mnemonic FAST HUG. A cross-sectional and descriptive study was carried out, with qualitative and quantitative assessment, using a probabilistic sample. Data collection was performed through an online questionnaire with objective questions approved by the Ethics and Research Committee - CEP. Of the 50 ICU professionals contacted, only 25 were willing to respond to the consultation. Of these, 52\% said they knew about the FAST HUG checklist. The best time to apply the protocol proved to be during the Rounds. Other relevant information found showed that $40 \%$ of professionals had difficulties in applying the checklist items, evidencing the need for training to align ideas and
\end{abstract}


conduct the work. The institution of Rounds in the ICUs proves to be an essential utility for the application of the mnemonic FAST HUG.

Keywords: Clinical protocols; Intensive Care Unit; Multi-professional; Checklist; Bundles.

\begin{abstract}
Resumen
Este estudio tiene como objetivo verificar el conocimiento de los profesionales de la salud, médicos, enfermeras y fisioterapeutas del SUS, que laboran en la Unidad de Cuidados Intensivos de un hospital público del norte de Brasil, sobre el mnemónico FAST HUG. Se realizó un estudio descriptivo, transversal, con valoración cualitativa y cuantitativa, utilizando una muestra probabilística. La recolección de datos se realizó a través de un cuestionario en línea con preguntas objetivas aprobadas por el Comité de Ética e Investigación - CEP. De los 50 profesionales de UCI contactados, solo 25 estuvieron dispuestos a responder a la consulta. De estos, el $52 \%$ dijo que conocía la lista de verificación FAST HUG. El mejor momento para aplicar el protocolo resultó ser durante las Rondas. Otra información relevante encontrada mostró que el $40 \%$ de los profesionales tenían dificultades para aplicar los ítems de la lista de verificación, evidenciando la necesidad de capacitación para alinear ideas y realizar el trabajo. La institución de las Rondas en las UCI resulta ser una utilidad fundamental para la aplicación del mnemónico FAST HUG.

Palabras clave: Protocolos clínicos; Unidad de Terapia Intensiva; Multiprofesional; Lista de Verificación; Manojos.
\end{abstract}

\title{
1. Introdução
}

A unidade de terapia intensiva (UTI) é uma área crítica, designada a internação de pacientes graves, que necessitam de atenção da equipe de forma constante, onde uma falta de atenção ou erros médicos podem causar complicações ou até mesmo levar o paciente à morte (Segato, 2021). Com o objetivo de aperfeiçoar o atendimento ao paciente crítico, o médico Jean-Louis Vincente criou o mnemônico FAST HUG, uma sigla em inglês, que aponta sete aspectos essenciais do cuidado ao paciente crítico no geral (Santos, et al., 2017). Vincent JL (2005) propôs o conceito "O abraço rápido ", uma relação que destaca sete aspectos indispensáveis no atendimento ao paciente crítico, que devem ser analisados ao menos uma vez ao dia. O FAST HUG é uma sigla em Inglês na qual F é de Feeding (Alimentação), A de Analgesia, S de Sedation (Sedação), T de Thromboembolic Prophylaxis (Profilaxia de trombose venosa), H de Head of the Bed Elevated (Cabeceira do leito elevada), $\mathrm{S}$ de Stress Ulcer Prevention (Prevenção de úlcera de estresse), G de Glucose control (Controle Gligêmico). (Ghiggi e Almeida, 2021).

Feeding (Alimentação): em pessoas idosas, que são mais prevalentes na UTI, os efeitos catabólicos da doença causada pelos distúrbios nutricionais, tem conexão com a piora do quadro clínico, aumenta por exemplo a incidência de lesões por pressão. O estado nutricional afeta diretamente a evolução clínica, como redução da morbimortalidade, redução das reações catabólicas, fortalecimento do sistema imunológico, manutenção da integridade funcional do trato gastrointestinal e redução do tempo de internação na unidade de terapia intensiva (UTI). Diante desse cenário, é muito importante a terapia nutricional adequada, seja ela por via oral, enteral ou parenteral (Fischer, et al., 2020; Volkert, et al, 2019).

Analgesia: a dor aflige de forma psicológica e fisiológica, devido não somente a sua doença como também a procedimentos realizados na rotina. $\mathrm{O}$ alívio adequado da dor deve ser examinado rotineiramente, pacientes enfermos podem ser difíceis de expressar sua dor, pois a maioria está inconsciente, havendo então a necessidade de avaliar sinais relacionados a expressões faciais, movimentos, frequência cardíaca e pressão arterial. Os opioides como a Fentanila e Morfina, são os mais usados na terapia intensiva, em infusões contínuas ou em bolus, quando necessário (Vorpagel, et al., 2021; Gomes, et al., 2019).

Sedation (Sedação): a sedação adequada é essencial para pacientes criticamente enfermos que precisam de tratamento com ventilação mecânica. A sedação profunda pode levar a algumas consequências indesejáveis, como aumento do tempo na ventilação mecânica, podendo causar escaras, alterações na memória e aumento da mortalidade. Quando a sedação é inadequada causa dor, agitação, delírio. Na UTI, os fármacos mais utilizados para sedação são os benzodiazepínicos em 
conjunto com analgésicos opioides. O controle adequado com escalas para titulação da sedação, como a escala de RASS, auxiliam no ajuste de dose, reduzindo o tempo de internação e eventos adversos (Figueira, et al., 2020; Silva, et al, 2017).

Thromboembolic Prophylaxis (Profilaxia de trombose venosa): pacientes críticos apresentam diversos fatores de risco para desenvolvimento da trombose, tais como: traumas, cirurgias, neoplasias, e o fato de ficarem restritos ao leito, aumentando a possibilidade de eventos graves secundários (Ghiggi e Almeida, 2021). Jiménez, et al., (2019) afirma que a doença tromboembólica venosa (TEV) é um dos principais distúrbios causados em pacientes hospitalizados, sendo constituído por trombose venosa profunda e embolia pulmonar. A trombose venosa profunda (TVP) é uma doença causada por várias condições, caracterizada pela formação de trombos em veias profundas que podem surgir de forma súbita. A profilaxia pode ser feita através de ações farmacológicas ou não farmacológicas como meias de compressão pneumáticas e a utilização de heparinas (Oliveira, et al., 2021).

Head of the Bed Elevated (Cabeceira do leito elevada): a pneumonia aspirativa é um evento adverso que acomete paciente internado em unidade de terapia intensiva, causada pelas secreções das vias aéreas superiores ou refluxo do trato gastrointestinal. A elevação da cabeceira do leito em $30^{\circ}$ e $45^{\circ}$ é considerada uma medida simples, que não gera custos adicionais e é uma das orientações para prevenir broncoaspiração, aumentar o volume corrente inspirado, reduzir o esforço muscular e a reduzir a taxa de atelectasia (Branco, 2020; Fernandes, et al., 2021; Silva, et al, 2021).

Stress Ulcer Prevention (Prevenção de úlcera de estresse): pacientes críticos correm risco de desenvolver erosões da mucosa gastrointestinal, pois estão propensas a situações estressantes como ventilação mecânica e polimedicamentos (Freitas e Araújo, 2021). Por isso, a importância de se iniciar a prevenção, sendo uma parte importante do tratamento, onde os medicamentos mais utilizados são os inibidores da bomba de prótons, como o Pantoprazol (Santos, 2020).

Glucose control (Controle Glicêmico): a glicose é um carboidrato, é ele que fornece energia para o corpo, sendo necessário para o bom funcionamento do organismo. Quando os mecanismos de controle da glicose se alteram, podem elevar ou diminuir a glicemia, causando comprometimento nas funções do organismo. O índice de mortalidade e de tempo de internação são elevadas em pacientes graves com glicemia aumentada ou diminuída (Granadeiro, et al., 2020; Segato, 2021).

Uma forma de atendimento integralizado, que são essenciais ao cuidado do paciente crítico, são as equipes multidisciplinares, na qual médicos, enfermeiros, fisioterapeutas, farmacêuticos clínicos, psicólogos e outros profissionais, contribuem para os cuidados intensivos nas rodadas multidisciplinares (Rounds) a beira leito, que são realizados diariamente com verificações e metas diárias para garantir resultados positivos para o paciente (Borges et al., 2021; Lee, et al., 2019).

Para cada profissional existem protocolos, bundles e checklists padronizados, o que facilita o acompanhamento terapêutico do paciente, como checklist para passagem de acesso venoso central e checklist na posição prona, além disso, para um melhor suporte ao paciente crítico, é necessário a aplicação do checklist FAST HUG, que contém aspectos essenciais do cuidado ao paciente, método simples, evitando erros, reduzindo as complicações dentro da Unidade de Terapia Intensiva (Pinho, et al., 2020; Brito, 2021).

A realização de bundles e checklists são valorosas para a qualidade do atendimento baseado em evidências científicas, que tenha resultado positivo no paciente crítico, reduzindo a mortalidade, complicações como pneumonia associada à ventilação mecânica e úlcera de estresse, reduz dias de permanência na unidade de terapia intensiva e desta forma, reduzindo custos. A aplicação do protocolo permite cuidados de boa qualidade, seguro, eficaz, rentável, que não acarreta em custos e não se restringe a nenhum grupo de pacientes e ainda auxilia na tomada de decisões clínicas adequadas (Jiménez, 2019; Silva e Almeida, 2020).

Por isso, a importância da aplicação do mnemônico na rotina da UTI, pois melhora a qualidade do atendimento ao paciente crítico, não se limita a nenhum grupo específico de pacientes, auxilia nas decisões clínicas da equipe multidisciplinar e na segurança destes. 
O presente estudo tem como objetivo verificar o conhecimento dos profissionais médicos, enfermeiros e fisioterapeutas que atuam na unidade de terapia intensiva (UTI), a respeito do mnemônico FAST HUG, em um hospital público da região norte brasileira.

\section{Metodologia}

Foi realizado um estudo do tipo transversal e descritivo, estabelecendo relações entre variáveis, envolvendo o uso de técnicas padronizadas para coleta de dados e avaliação qualitativa e quantitativa, visando determinar o número de ocorrências, as intensidades ou as modalidades dos fenômenos individuais que compõem o fenômeno coletivo (Gil, 1991).

A amostra é do tipo probabilística, onde foram convidados a participar da pesquisa como critério de inclusão, apenas médicos, enfermeiros e fisioterapeutas estatutários, emergenciais e residentes atuantes da terapia intensiva.

A coleta de dados foi realizada por meio de questionário online, com questões objetivas, aprovado pelo Comitê de ética e pesquisa - CEP, com o número CAAE 43743221.4.0000.5298, aplicado a médicos, enfermeiros e fisioterapeutas da Unidade de Terapia Intensiva adulto de um hospital público da região norte brasileira, que aceitaram participar da pesquisa, assinando o termo de consentimento livre e esclarecido, através da ferramenta online Google Forms. Os dados dos questionários foram transcritos para planilha em Programa MS Excel Office. Posteriormente, estes foram preparados através do programa BIOESTAT 5.0 para a realização da análise estatística descritiva e também para a análise estatística inferencial, usando-se o teste do Qui-Quadrado e o teste Exato de Ficher com nível de significância p < 0,05.

\section{Resultados e Discussão}

Foram convidados a participar da pesquisa 50 profissionais, porém, apenas 25 destes que atuam na unidade de terapia intensiva em um hospital público da região norte do Brasil aceitaram. Destes 11 enfermeiros (44,0\%) (6 residentes, 3 estatutárias e 2 emergencial), 11 fisioterapeutas (44,0\%) (5 residentes, 4 estatutários e 2 emergencial) e 3 médicos (12,0\%) sendo todos os três emergenciais. A maioria dos profissionais, 16 (64,0\%) possuíam idade entre 26 e 40 anos, $8(32,0 \%)$ tinham no máximo 25 anos e apenas $1(4,0 \%)$ possuía idade superior a 40 anos de idade. Ao que se refere ao tempo de formação foi identificado que 17 deles, (68,0\%) tem menos de 5 anos de formação, $3(12,0 \%)$ de 6 a 10 anos e 5 (20,0\%) possuem 10 ou mais anos de formação (Tabela 1). 
Research, Society and Development, v. 10, n. 16, e41101623502, 2021

(CC BY 4.0) | ISSN 2525-3409 | DOI: http://dx.doi.org/10.33448/rsd-v10i16.23502

Tabela 1 - Caracterização dos profissionais participantes da pesquisa. Cacoal/RO, 2021.

\begin{tabular}{lcc}
\hline Profissão & $\mathbf{N}$ & $\%$ \\
\hline Enfermeiro & 11 & 44,0 \\
Fisioterapeuta & 11 & 44,0 \\
Médico & 3 & 12,0 \\
Total & 25 & 100,0 \\
\hline Vínculo & $\mathbf{N}$ & $\%$ \\
\hline Residente & 11 & 44,0 \\
Estatutário & 7 & 28,0 \\
Emergencial & 7 & 28,0 \\
Total & 25 & 100,0 \\
\hline Idade & $\mathbf{N}$ & $\%$ \\
\hline De 26 a 40 anos & 16 & 64,0 \\
Até 25 anos & 8 & 32,0 \\
Acima de 41 anos & 1 & 4,0 \\
Total & 25 & 100,0 \\
\hline Tempo de Formação & $\mathbf{N}$ & $\% 8,0$ \\
\hline 1 a 5 anos & 17 & 12,0 \\
6 a 10 anos & 3 & 20,0 \\
10 anos ou mais & 5 & 100,0 \\
\hline Total & 25 & \\
\hline
\end{tabular}

Fonte: Zumack (2021).

Como parte fundamental deste estudo, perguntamos a estes profissionais quanto ao conhecimento e à importância da aplicação do checklist que contemple os 7 itens do FAST HUG na rotina da UTI, visando a melhoria da qualidade do atendimento ao paciente crítico. Destes, 13 (52,0\%) disseram ter conhecimento do checklist e os demais 12 (48,0\%) não possuíam este conhecimento.

No entanto, quando questionados sobre quais ações eles realizam com o paciente em seu dia a dia, mesmo sem conhecimento dos itens contidos no mnemônico FAST HUG, boa parte deles os realizam diariamente, alguns inclusive com uma frequência muito elevada como a verificação de sedação e verificação de cabeceira elevada, realizadas por $92,0 \%$ dos profissionais, seguidos por verificação de analgesia (84,0\%) e verificação de prevenção de úlcera de estresse (80,0\%) (Figura 1). 
Figura - Ações constantes no protocolo FAST HUG desenvolvidas pelos profissionais participantes da pesquisa. Cacoal/RO, 2021 .

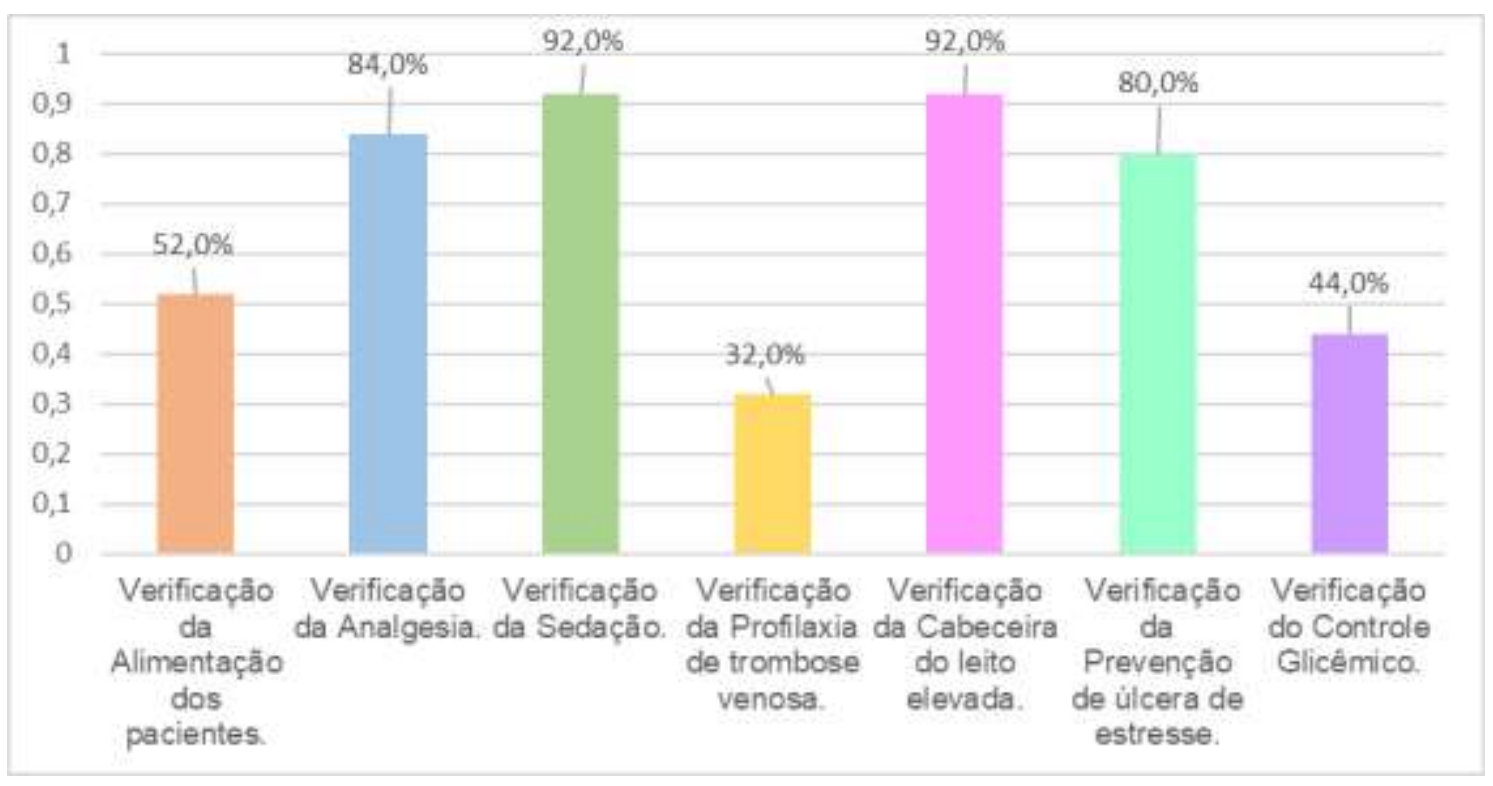

Fonte: Zumack (2021).

De acordo com Jiménez, et al., (2019), que fez um estudo para determinar a redução da mortalidade com a aplicação dos itens contidos no FAST HUG, onde mostrou que teve redução dos riscos de mortalidade quando pelo menos 3 itens são aplicados no dia a dia, como a alimentação, sedação e tromboprofilaxia.

O item menos observado no dia a dia, foi a verificação da profilaxia de trombose venosa (32\%), corroborando com o estudo de Lopes, et al., (2017), onde demonstrou que 34,29\% dos pacientes analisados não receberam profilaxia para TEV.

O estudo de Sousa, et al., (2018), identificou que protocolos de monitoramento da glicemia é importante para assistência, referindo como um facilitador para a acompanhamento, porém alguns entrevistados relataram dificuldade em executar o protocolo, sendo necessário melhor orientação.

Mesmo aplicando estes itens em seu dia a dia, 10 (40,0\%) dos profissionais entrevistados disseram possuir alguma dificuldade na aplicação dos mesmos, enquanto os demais $15(60,0 \%)$ alegaram não possuir dificuldade alguma. Entre os que disseram possuir dificuldade na aplicação dos itens, os mesmos disseram que isso se dá por falta de treinamento (68,0\%), ausência de protocolo $(64,0 \%)$ ou por falta de tempo para uma aplicação adequada em 28,0\% das respostas. Corroborando com esse resultado, o estudo de Faria e Souza (2021), que fez uma revisão descritiva da literatura, mostrou que 38,4\% dos artigos analisados tiveram dificuldade de implementação dos bundles de prevenção.

Também foi questionado sobre a existência de um protocolo para aplicação destes itens, uma vez que eles fazem parte da rotina diária dos mesmos. Entre os entrevistados, 10 (40,0\%) deles disseram não haver nenhum protocolo, 9 (36,0\%) disseram existir um protocolo para essa aplicação, enquanto $6(24,0 \%)$ disseram não ter conhecimento da existência de protocolo. Esses dados apresentam diferenças estatisticamente significante $(\mathrm{p}<0,05)$, uma vez que a não existência de protocolo pode levar a um não conhecimento dos itens constantes no checklist do mnemônico FAST HUG (Tabela 2), mostrando a importância da capacitação dos profissionais para atuarem na unidade de terapia intensiva, como mostra o trabalho de Legal, et al. (2018). 
Tabela 2 - Distribuição dos profissionais participantes da pesquisa com relação ao conhecimento do checklist e a existência de protocolo para aplicação dos itens. Cacoal/RO, 2021.

\begin{tabular}{|c|c|c|c|c|c|}
\hline \multirow{3}{*}{ Existe Protocolo } & \multicolumn{4}{|c|}{ Conhece checklist } & \multirow{3}{*}{$\begin{array}{c}\text { Teste Exato de } \\
\text { Fisher } \\
\text { P Value }\end{array}$} \\
\hline & \multicolumn{2}{|c|}{ Sim } & \multicolumn{2}{|c|}{ Não } & \\
\hline & $\mathbf{n}$ & $\%$ & $\mathbf{n}$ & $\%$ & \\
\hline Sim & 4 & 30,8 & 5 & 41,7 & \multirow{4}{*}{0,049} \\
\hline Não & 8 & 61,5 & 2 & 16,7 & \\
\hline Não sei & 1 & 7,7 & 5 & 41,7 & \\
\hline Total & 13 & $52.0 \%$ & 12 & $48.0 \%$ & \\
\hline
\end{tabular}

Fonte: Zumack (2021).

Embora não tenha apresentado diferenças estatisticamente significantes ( $p>0,05)$, é possível verificar que entre os 10 (40,0\%) profissionais que apresentam alguma dificuldade em aplicar os itens, destes, 6 (60,0\%) responderam não ter conhecimento dos itens do FAST HUG. Percebe -se que a maioria $(60,0 \%)$ dos que responderam ter conhecimento do protocolo não apresentam dificuldades de aplicação dos itens em sua rotina diária (Tabela 3 ).

Tabela 3 - Distribuição dos profissionais participantes da pesquisa com relação ao conhecimento do checklist e a dificuldade de aplicação dos itens. Cacoal/RO, 2021.

\begin{tabular}{|c|c|c|c|c|c|}
\hline \multirow{3}{*}{$\begin{array}{l}\text { Tem dificuldade em } \\
\text { aplicar os itens }\end{array}$} & \multicolumn{4}{|c|}{ Conhece checklist } & $\begin{array}{c}\text { Teste Exato de } \\
\text { Fisher }\end{array}$ \\
\hline & \multicolumn{2}{|c|}{ Sim } & \multicolumn{2}{|c|}{ Não } & \multirow[b]{2}{*}{ P Value } \\
\hline & n & $\%$ & n & $\%$ & \\
\hline Sim & 4 & 40,0 & 6 & 60,0 & \multirow{3}{*}{0,428} \\
\hline Não & 9 & 60,0 & 6 & 40,0 & \\
\hline Total & 13 & $52.0 \%$ & 12 & $48.0 \%$ & \\
\hline
\end{tabular}

Fonte: Zumack (2021).

Foi possível verificar portanto, que para 15 (60,0\%) profissionais, o melhor momento para a aplicação dos itens do mnemônico FAST HUG é durante o round, já 9 (36,0\%) acham que o melhor momento é durante a avaliação do paciente e apenas $1(4,0 \%)$ afirmou ser na troca de plantão o melhor momento. Quanto ao conhecimento de identificar o melhor momento de aplicação dos itens do protocolo FAST HUG e o conhecimento do mesmo, não se observou diferenças estatisticamente significantes $(p>0,05)$ mostrando que o conhecimento do checklist não influencia na tomada de decisão do momento em se aplicar os itens (Tabela 4). 
Tabela 4 - Distribuição dos profissionais participantes da pesquisa com relação ao conhecimento do checklist e o momento adequado a aplicação dos itens. Cacoal/RO, 2021.

\begin{tabular}{|c|c|c|c|c|c|}
\hline \multirow{3}{*}{ Momento adequado a FAST HUG } & \multicolumn{4}{|c|}{ Conhece checklist } & \multirow{3}{*}{$\begin{array}{c}\text { Teste Exato de } \\
\text { Fisher } \\
\text { P Value }\end{array}$} \\
\hline & \multicolumn{2}{|c|}{ Sim } & \multicolumn{2}{|r|}{ Não } & \\
\hline & $\mathbf{n}$ & $\%$ & $\mathbf{n}$ & $\%$ & \\
\hline Durante o Round & 7 & 46,7 & 8 & 53,3 & \multirow{4}{*}{0,411} \\
\hline Durante a avaliação do paciente & 6 & 66,7 & 3 & 33,3 & \\
\hline Na passagem de plantão & 0 & 0,0 & 1 & 100,0 & \\
\hline Total & 13 & 52,0 & 12 & 48,0 & \\
\hline
\end{tabular}

Fonte: Zumack (2021).

O estudo de Barcellos e Chatkin (2020), fez a implantação de uma lista de verificação para redução do tempo de ventilação mecânica, sendo que o melhor momento foi durante os Rounds com a equipe multiprofissional, mostrando a redução no tempo de internação dos pacientes na UTI, melhorando a aceitação nos processos de cuidado, além de melhorar a comunicação entre a equipe, mostrando ainda que a participação da equipe multiprofissional na implantação do checklist apresenta resultados positivos.

O trabalho de Guzinski (2019), mostrou que a experiência com os rounds multidisciplinares promoveu a comunicação assertiva a respeito das práticas do dia a dia, permitindo a participação e colaboração de todos os profissionais para solucionar dúvidas e problemas que possam surgir, associado a práticas baseadas em evidências, também podendo ocorrer a programação da possível alta desse paciente, sendo observado então a redução no tempo de internação e redução de lesões por pressão.

\section{Conclusão}

A análise desse estudo nos levou a observar que apenas $52 \%$ dos profissionais respondentes conheciam o checklist apresentado, sendo que os $48 \%$ que não conheciam esse procedimento aplicavam um ou outro item desse checklist na sua rotina normal de trabalho. Também constatamos que $40 \%$ dos que praticam na íntegra o checklist apresentam dificuldades em realizar os itens constantes. A sedação e verificação da cabeceira do leito elevada foram os itens mais avaliados, enquanto o menos analisado foi a profilaxia de trombose venosa. $40 \%$ dos respondentes mostraram ter dificuldade de aplicar corretamente os itens diariamente. $64 \%$ dos entrevistados afirmaram que a incorreta aplicação do protocolo se dá por falta de treinamento A considerar que os referidos protocolos ao ser implementados, complementarmente deverão ser realizadas capacitações e educação continuada para o alinhamento e compreensão da linha de conduta junto aos aplicadores do protocolo.

Houve grande divergência nas respostas, quanto à existência do protocolo implementado, tendo a maioria questionado a própria existência do referido protocolo. A concluir que a falta de capacitação na implementação de protocolos dificulta a execução de qualquer programa e um processo de capacitação de toda a equipe unificaria um proceder de melhor atendimento, uma maior credibilidade no sistema de assistência ao paciente.

Quanto ao melhor momento para a execução do mnemônico foi demonstrado ser durante os Rounds, desenvolvido com a equipe multiprofissional, sugerindo que esses Rounds virem rotina "obrigatória” nas Unidades de Terapia Intensiva, associada a práticas baseadas em evidência.

Após a implementação do mnemônico FAST HUG, sugere-se um novo estudo para realização do mnemônico FAST HUG-MAIDENS, um checklist clínico com intervenções farmacêuticas visando a otimização da farmacoterapia dos pacientes internados na unidade de terapia intensiva. 


\section{Referências}

Barcellos, R. A. \& Chatkin, J. M. (2020). Impacto de uma lista de verificação multidisciplinar na duração da ventilação mecânica invasiva e no tempo de permanência na UTI. Jornal brasileiro de pneumologia: publicação oficial da Sociedade Brasileira de Pneumologia e Tisilogia, 46 (3), e20180261.

Borges, M. L., Caruso, P., \& Nassar, A. P. (2021). Carga de trabalho da enfermagem associada com frequência de visitas multidisciplinares: um estudo transversal. Revista Brasileira de terapia intensiva, 33 (1), 82-87.

Branco, A., Lourençone, E. M. S., Monteiro, A. B., Fonseca, J. P., Blatt, C. R., \& Caregnato, R. C. A. (2020). Educação para prevenção da pneumonia associada à ventilação mecânica em unidade de terapia intensiva. Revista Brasileira de Enfermagem, 73.

Brito, A. M. D. (2021). Análise de intervenções farmacêuticas utilizando um instrumento de acompanhamento farmacêutico em uma unidade de terapia intensiva pediátrica. Trabalho de conclusão de especialização, Universidade Federal do Rio Grande do Sul, Hospital de Clínicas de Porto Alegre, Porto Alegre/RS. $56 \mathrm{f}$.

Faria, E. A de P., \& Souza, R. D. de (2021). Utilização de bundles para prevenção de pneumonia associada à ventilação (PAV). Trabalho de conclusão de curso (TCC) em Enfermagem, PUC Goiás.

Fernandes, B. C., Araújo, A. M. de B., da Silva, N. L., Tanaka, L. H. V. B., \& Yoshikawa, C. A. (2021). Medidas preventivas para diminuição no risco de pneumonia associada à ventilação mecânica. Revista Pub Saúde. 6,133.

Figueira, L. A. F., Mendes, L. C., Mulaski, L. S., Vieira, L. A. P., da Silva, M. M., Alves, M. L. C. D., \& Medeiros, D. N. (2020). Sedação de pacientes na unidade de terapia intensiva. Cadernos da Medicina-UNIFESO, 2(3).

Fischer, M. de Q., Baelz, K., Dalmonte, K. S., \& Poll, F. A. (2020). Relação entre o estado nutricional, dieta e diagnóstico de idosos internados em unidade de terapia intensiva. Estudos Interdisciplinares sobre o Envelhecimento, 25(2).

FREITAS, D. S., \& ARAÚJO, K. S. B. (2021). As indicações da profilaxia de úlceras gástricas em pacientes pediátricos hospitalizados: uma revisão integrativa. Trabalho de conclusão de curso (TCC) em medicina, Repositório institucional Tiradentes.

Ghiggi, K. C., \& Almeida, G. B. (2021). Rotinas de Unidades de Terapia Intensiva. VITTALLE-Revista de Ciências da Saúde, 33(1), $185-202$.

Gil, A.C. (1991). Métodos e Técnicas de Pesquisa Social. Atlas.

Gomes, R. da S., de Carvalho, A. M. R., Medeiros, D. da S., \& Cavalcante, M. G. (2019). Perfil farmacoterapêutico dos pacientes críticos de um hospital privado de alta complexidade. Journal of Applied Pharmaceutical Sciences, (6): 159-167

Granadeiro, R. M. de A., Sá, S. P. C., Christóvam, B. P., Spezani, R. dos S., \& Granadeiro, D. da S. (2020). Hipoglicemia durante a infusão contínua de insulina na Unidade de Terapia Intensiva. Research, Society and Development, 9(9), e163996748-e163996748.

Guzinski, C., Lopes, A., Flor, J., Migliavaca, J., Tortato, C., \& Pai, D. D. (2019). Good practices for effective communication: the experience of the interdisciplinary round in orthopedic surgery. Boas práticas para comunicação efetiva: a experiência do round interdisciplinar em cirurgia ortopédica. Revista gaúcha de enfermagem, 40(spe), e20180353.

Jiménez, B.B., Jiménez, C.C., Marines, L. A. R., \& Rodríguez, M. M. (2019). Aplicação do protocolo FAST-HUG e sua associação com a mortalidade do paciente crítico na UTI. Medicina crítica (Colegio Mexicano de Medicina Crítica), 33(3), 130-138.

Lee, H., Ryu, K., Sohn, Y., Kim, J., Suh, G. Y., \& Kim, E. (2019). Impact on Patient Outcomes of Pharmacist Participation in Multidisciplinary Critical Care Teams: A Systematic Review and Meta-Analysis. Critical care medicine, 47(9), 1243-1250.

Legal, D., \& Medeiros, K. D. D. (2018). Conhecimento da enfermagem sobre a prevenção da pneumonia associada à ventilação mecânica num hospital público. Revista de Atenção à Saúde (ISSN 2359-4330), 16(57).

Lopes, B. A. C., Teixeira, I. P., Souza, T. D. D., \& Tafarel, J. R. (2017). Sabemos prescrever profilaxia de tromboembolismo venoso nos pacientes internados? Jornal Vascular Brasileiro, 16, 199-204.

Oliveira, A., Valença, M. M., Libânia, I. A., \& Cruz, F. (2021). Profilaxia de tromboembolismo venoso para pacientes hospitalizados. Avanços em Medicina, 45-50.

Pinho, C. M., Bezerra, B. L., de Lima, A. B. A., Silva, D. A. V., da Silva, E. L., Reis, J. D. A. de O., \& de Lima, M. C. L. (2020). O uso dos bundles em unidades de terapia intensiva: prevenção e redução das infecções. Rev. Enferm. Digit. Cuid. Promoção Saúde. 2020;5(2):117-124

Santos, R. R., Henrique, D. de M., de Almeida, L. F., Penteado, M. de S., Pereira, S. R. M., \& Santos, D. P. da S. (2017). Fast hug: um aliado na manutenção diária dos cuidados de enfermagem ao paciente crítico. Enfermagem em Foco, 8(1), 57-61.

Santos, Y. D. A. P. D., Younes-Ibrahim, M. S., Crozatti, L. L., Raglione, D., Cardozo, L. C. M., Besen, B. A. M. P., \& Mendes, P. V. (2020). Adesão a um protocolo de profilaxia de úlcera de estresse em pacientes críticos: estudo de coorte prospectiva. Revista Brasileira de Terapia Intensiva, 32, 37-42.

Segato, T. H. F. (2021). Solução orientada a agentes inteligentes para controle glicêmico de pacientes internados em UTI. Dissertação (Mestrado em Informática)-Universidade de Brasília.

Silva, A. S., \& Almeida, B. L. D. (2020). Bundle e Checklist aplicado a área da saúde: Uma análise conceitual. Trabalho de conclusão de curso (TCC) em Enfermagem, PUC Goiás.

Silva, D. C. D., Barbosa, T. P., Bastos, A. S. D., \& Beccaria, L. M. (2017). Associação entre intensidades de dor e sedação em pacientes de terapia intensiva. Acta Paulista de Enfermagem, 30, 240-246. 
Research, Society and Development, v. 10, n. 16, e41101623502, 2021

(CC BY 4.0) | ISSN 2525-3409 | DOI: http://dx.doi.org/10.33448/rsd-v10i16.23502

Silva, M. F. de O., Campara, M. A. S., da Silva, F. V. R., Figueiredo, P. T. de S., \& Campolino, R. G. (2021). Impactos da implementação do Bundle de pneumonia associada à Ventilação Mecânica: Manutenção da cabeceira da cama elevada de $30^{\circ}$ a $45^{\circ}$. Brazilian Journal of Health Review, 4(2), 7381-7386.

Sousa, T. L., Matos, E., \& Salum, N. C. (2018). Indicativos para melhores práticas no controle glicêmico em unidade de terapia intensiva. Escola Anna Nery, 22.

Vincent, J. L. (2005). Give your patient a fast hug (at least) once a day. Critical care medicine, 33(6), 1225-1229.

Volkert, D., Beck, A. M., Cederholm, T., Cruz-Jentoft, A., Goisser, S., Hooper, L. \& Bischoff, S. C. (2019). ESPEN guideline on clinical nutrition and hydration in geriatrics. Clinical nutrition, 38(1), 10-47.

Vorpagel, K. M., Schein, J. L., \& Sangoi, K. C. M. (2021). Avaliação da dor no paciente internado em unidade de terapia intensiva: relato de experiência. In Congresso Internacional em Saúde (No. 8). 